\title{
A novel algorithm for multi-node load forecasting based on big data of distribution network
}

\author{
Guangsong $\mathrm{Hou}^{1}, \mathrm{Ke} \mathrm{Xu}^{1}$, Shoubin $\mathrm{Yin}^{1}$, Yang Wang ${ }^{1}$, Yan $\mathrm{Han}^{1}$, Zhiguo Wang $^{1}$, Yifei Mao ${ }^{2, \mathrm{a}}$ and \\ Zhengxin Lei ${ }^{2}$ \\ ${ }^{1}$ State Grid Shandong electric power company Heze power supply company, Heze, 274000, China \\ ${ }^{2}$ Key Laboratory of Control of Power Transmission and Conversion, Ministry of Education, Shanghai Jiao Tong \\ University, Shanghai, 200240, China
}

\begin{abstract}
Effective load forecasting for different scales of loads is essential for the planning and operation of distribution network. The extending scale of the network, the diversity of load types and the rapid increase of data volume are some of the facing problems. In this paper, we propose a novel method for multi-node load forecasting, AR-ANN, which takes those problems into consideration. This new algorithm makes a combination of AR method and BP neural network method while eliminating their disadvantages. Comparing to traditional bottom-up method, a top-down method is more applicable when considering the limitation of measurement equipment in distribution network. Both top-down method and bottom-up method are tested in this paper by using AR-ANN algorithm. The data processing speed and the forecasting accuracy of AR-ANN is validated by several tests: an ordinary single-node load forecast, two multi-node load forecasts by traditional bottom-up method and by the new top-down method.
\end{abstract}

Keywords: Multi-Node Load Forecast; BP-ANN; AR; big data; distribution network.

\section{Introduction}

Because of the increasing load of China and the modification of the distribution network structure, different kinds of system analysis, which mainly consider load flow analysis, N-1 contingency analysis and emergency power supply, should have more detailed data to support. Thus multi-node load forecasting for changeable distribution networks based on big data is needed. Traditional multi-node load forecasting can reduce the prediction error due to the more characteristically obvious nodes compared to the whole. Nowadays, the load of active users becomes more random with the insert of different new kinds of power generating and consuming devices, thus load forecasting of some terminal nodes and users is particularly emphasized. Meanwhile, the developing distribution network involves wide range and big volume of data, which makes fast data processing important in both traditional and big data-based load forecasting; while additional installation of measurement equipment is economically unfavorable, it is necessary to forecast load by using the existing data from substations, electric power lines and smart meters.

Researchers have been doing some optimization and modification to the classical multi-node load forecasting method based on distributed computing theory. An improved methodology based on general

a Corresponding author : fiomeiya@sjtu.edu.cn

(C) 2016. The authors - Published by Atlantis Press 
regression neural networks for short-term multi-node load forecasting is introduced in [1] and it also proposed another method which aims to limit calculation time by reducing input variables. Reference [1] compared the two methods with traditional general regression neural networks by tests on a New Zealand distribution subsystem. Additionally, the author divided the methods into local load forecasting, global load forecasting and participation factor forecasting. Reference [2] constructed a framework of self-adapting dynamic load models of ultra-short-term forecasting for multi-node active and reactive load based on the ideas of "hierarchy" and sub-area. Reference [3] used fuzzy-rough sets to determine the initial weights of artificial neural networks for short term load forecasting in order to select the most significant input variables.

Among the improved conventional forecasting techniques, the stochastic time series approach is most popular[4] because of its accuracy. AR (Auto-Regressive Model), one of the time series analysis approach characterized by added linear filter, has high short-term forecasting accuracy however is unable to process data of multiple sources. On the other hand, artificial intelligence techniques have been much studied these years to apply for load forecasting. The models that have received the largest share of attention are the artificial neural networks (NNs)[5]. A great number of papers have done practical tests with these nonlinear algorithms and compared the results. Typical NN methods are BP-ANN (Back Propagation Artificial Neural Network), ELMAN Neural Network, FNN (Fuzzy Neural Network) and WNN (Wavelet Neural Network). A recent study of these four NN method compared their forecasting accuracy through different indicators[6]. The results of this study is shown in the table below.

Table 1. Performance comparison of four NN methods[6]

\begin{tabular}{|c|c|c|c|c|c|}
\hline \multirow{7}{*}{$\begin{array}{c}\text { Unprocessed } \\
\text { data }\end{array}$} & $\begin{array}{c}\text { Performance } \\
\text { index }\end{array}$ & BP-ANN & $\begin{array}{c}\text { ELMAN } \\
\text { NN }\end{array}$ & FNN & WNN \\
\cline { 2 - 6 } & $\begin{array}{c}\text { Mean squared } \\
\text { error } \\
\text { (MSE) (\%) } \\
\text { uare error } \\
\text { (RMSE) (\%) }\end{array}$ & 2.40933249 & 2.46387591 & 3.65791878 & 2.56212293 \\
\cline { 2 - 7 } & $\begin{array}{c}\text { Maximum } \\
\text { error (\%) }\end{array}$ & 6.94354001 & 7.49343331 & 8.85012490 & 7.98349630 \\
\hline \multirow{5}{*}{ Processed data } & $\begin{array}{c}\text { Mean squared } \\
\text { error } \\
\text { (MSE) (\%) }\end{array}$ & 2.23711067 & 2.23782612 & 2.78661130 & 2.03106810 \\
\cline { 2 - 7 } & $\begin{array}{c}\text { Root-mean-sq } \\
\text { uare error } \\
\text { (RMSE) (\%) }\end{array}$ & 2.66629658 & 2.73903932 & 3.32124424 & 2.58627537 \\
\cline { 2 - 7 } & $\begin{array}{c}\text { Maximum } \\
\text { error (\%) }\end{array}$ & 4.53485666 & 5.81497329 & 7.01129817 & 7.12242848 \\
\hline
\end{tabular}

We can see that BP-ANN has relatively better performance among theses NN methods both for unprocessed data and processed data. BP-ANN method can handle multi-source data but the prediction accuracy is affected by the setting of similar days. Considering the disadvantages of AR time series model and BP-ANN method, this paper proposes a load forecasting method, AR-ANN, which complements the advantages of AR and BP-ANN by adding AR into the training part of BP-ANN. For the load requirements, this paper presents a top-down multi-node big data based load forecasting method to face to the contradiction between the limitation of data collection and the need of node load forecasting of distribution network.

The main part of this paper is divided into three parts. The first part introduces load forecasting models and raises the new forecasting method. The second part mainly presents the AR method and 
BP-ANN method and the last part gives some tests of global load forecasting and two types of multi-node load forecasting by using AR-ANN method. The global load forecasting test validates the advantage of AR-ANN method in prediction error when comparing to BP-ANN method, and the traditional multi-node load forecasting test confirms that AR-ANN method is less time consuming than global load forecasting. In the third test where AR-ANN is applied to the new multi-node load forecasting, it is shown that the AR-ANN is more accurate than BP-ANN.

\section{Models of multi-node load forecasting}

According to the related papers[1-3]with author's own research experience, the definition of multi-node load forecasting can be summarized as follows: multi-node load forecasting is a distributed load forecasting in units of node which takes different sources of factors into consideration like the types of load, the electricity price and traffic condition. A node is a part of the network with common features (single user, whole of part of feeder line, substation etc.)

The "nodes" in the multi-node load forecasting are mutually exclusive and the set of the nodes forms the "whole". As shown in Figure 1, the structure of the nodes is branched from top to bottom and can be divided into several layers.

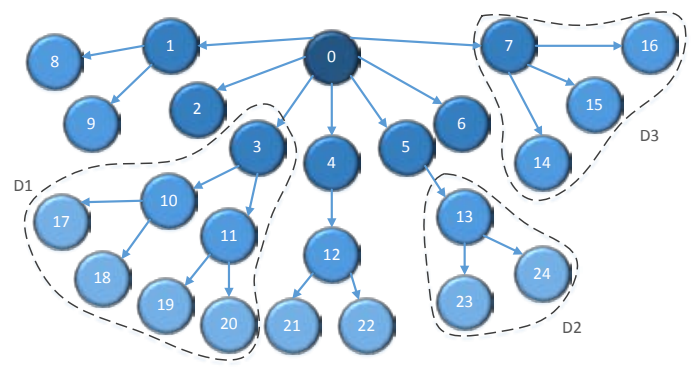

Figure 1. Structure of multi-node load forecast

In the actual load forecasting of distribution network, the structure is normally constructed by the layers of regional power grid, provincial power grid, main transformer line and the lower level voltage lines. The number of the arrows that lead to the node represents the layer of the node. Those lightest blue circles (node no.17 to no. 24) in the bottom represent the lines of $35 \mathrm{kV}$ and $10 \mathrm{kV}$. How to definite "whole" and "node" depends on the concrete forecasting problem. Take Figure 1 for example, we can choose those regional power grid as "whole" and the provincial power grid as "nodes". We can otherwise choose D1 - D3 parts as forecasting part so that those topmost nodes of these three parts are "wholes" and the others are "nodes".

Traditional methods mainly based on the bottom-up idea: start from the measured data of a single node, distributed forecast the changing load of the other loads, then get the whole load forecasting by integrating the results of all the nodes within. The mathematical expression can be summarized as:

$$
F\left(X_{1}, X_{2}, \ldots, X_{m}\right)=\eta \sum_{i=1}^{n} f\left(x_{i, 1}, x_{i, 2}, \ldots, x_{i, m}\right), i=1, \ldots, n
$$

where $F$ represents the forecasting algorithm of the whole and $\boldsymbol{X}_{1}, \ldots, \boldsymbol{X}_{\boldsymbol{m}}$ are the inputs of $F ; f$ represents the forecasting algorithm of the node and $\boldsymbol{x}_{i, 1}, \ldots, \boldsymbol{x}_{i, m}$ are its inputs; $i$ means the ith node in the network; $n$ is the number of nodes, $\eta$ is the ratio between the sum of the $f$ and $F$ due to the line loss or other reasons.

However, this bottom-up method might not be efficient in all cases. The data from the bottom or the terminal nodes of the distribution network can be incomplete or difficult to achieve in real-time due to lots of reasons like the independent data bases of different layers and permissions, the lack of measurement equipment or the instability of devices. Facing all this disadvantages, we proposes another method of multi-node load forecasting, "from the whole to the nodes", where the forecasting of the 
"whole" load can be an auxiliary manner to forecast the node load by use of the historical data of node loads when the real-time data of the nodes are unavailable. Its mathematical expression is as follows:

$$
f\left(x_{i, 1}, x_{i, 2}, \ldots, x_{i, m}\right)=\beta_{i} \cdot F\left(X_{1}, X_{2} \ldots, X_{m}\right), i=1, \ldots, n
$$

where $\beta_{i}$ represents the ratio of node load to global load diagonal matrix.

Since equations (1) and (2) specify neither the algorithm of load forecasting nor the time span of load forecasting, we now start modeling of the pre-day load forecasting by the AR-ANN method.

\subsection{Multi-node load forecasting by AR-ANN}

Load forecasts can be divided into three categories: ultra-short-term [7], short-term [8] and long-term forecasts [9]. Short-term forecasts are real-time forecasts which forecast every fifteen minutes. Short-term forecasts are usually from one hour to one day, and long-term forecasts are by month or by year. Pre-day load forecasting, which has a forecasting time span of 24 hours (from 0h to 24h), belongs to short-term forecasts though it forecasts every half hour or per hour which is more frequent. Thus short-term load forecasting methods can be applied to pre-day load forecasting and because of its forecasting frequency, lots of researches have been done in the selection of similar days [10-12]. Considering the need of rapid processing of big data, this paper simplifies the algorithm of selecting similar days and chooses experienced similar days which base on day type [13].

Load forecasting algorithms, according to how historical load derives to forecasting load, can be classified under extrapolation or correlation. ARMA [14], as one of the most common method of extrapolation, establishes a linear function of forecasting load, historical load and historical error. ANN (artificial neural networks) [15], as an example of correlation method, builds non-linear circuits between forecasting load, historical load, weather data and other multi-source factors. Load forecasting methods can otherwise be divided into statistical or artificial intelligence techniques. Statistical techniques include regression methods [16], time series, exponential smoothing model [17], Kalman filter based method [18], state estimate method [19]. Artificial intelligence techniques are such as ANN, fuzzy logic [16] and gray system theory[20].

The choice of similar days in this paper has already taken seasons and other attributes into consideration, so that the established time series forecasting model does not reflect seasonal variations. Under such condition, ARMA model takes only AR (auto-regressive) part and needs no MA (moving average) part [21]. Although AR method has high accuracy of ultra-short-term load forecasting, it cannot process data of multiple sources. Meanwhile the prediction error augments over time which becomes a disadvantage for pre-day forecasting. On the other hand, BP-ANN can handle multi-source data, however its prediction error is largely affected by the selection of similar days and network parameters. Comparing this two methods, we adjust the training inputs of BP-ANN by the results of AR model, which forms the novel algorithm AR-ANN proposed in this paper.

Figure 2 shows the basic flowchart of multi-node load forecasting applying AR-ANN algorithm.

The overall process contains mainly four steps: data reading, mode judgment and parameter setting, AR-ANN iteration and final result calculation with output display. In the step of data reading, the "whole", numbered 0, is the higher level node as described in Figure 1. In AR-ANN, both AR forecasting results and multi-source data of same period of time are used for training BP-ANN model, so that pre-day data of forecasting should be provided for data reading. Mode judgment and parameter setting decide the number of iterations and the formula with its parameters. To improve the operational

efficiency, we set $\eta$ in equation (1) to be 1 and let $\beta_{i}$ in equation (2) be the ratio diagonal matrix of similar days by chronological order. AR-ANN iteration could be either bottom-up which forecasts the load of node 0 to node $i$ by distributed computing, or top-down which calculates directly the global load only. Since AR-ANN is a modified algorithm of BP-ANN, the final result calculation follows the same way of BP-ANN which will be described further later in Section 3.2. 


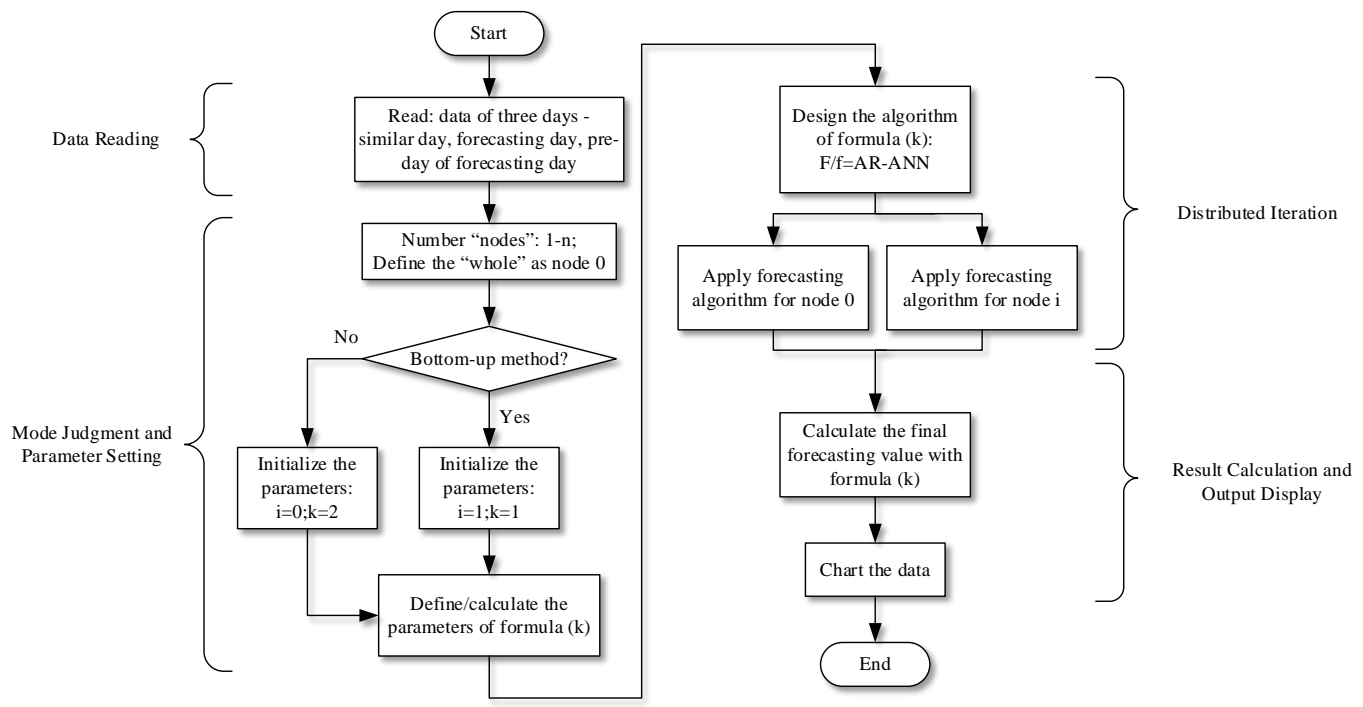

Figure 2. Flow chart of AR-ANN multi-node load forecast

The detailed AR-ANN based load forecasting process is as follows:

Step 1: Use time series AR algorithm to achieve the first predictive value of the forecasting day by the load curve of the day before. (The AR algorithm will be explained in Section 3.1.) For the non-pre-day load forecasting, the predictive value of AR algorithm will not still be the first point of the forecasting day since the real-time value is available during the prediction. We treat this situation as a short-term forecasting with increasing real-time data, which will be calculated in a test in Section 4.1.

Step 2: Set the predictive value of AR and the other sources of data (such as electricity price and weather data) as a new group of training sample points which replaces the time-corresponding group of training points in BP-ANN. Finish load forecasting by BP-ANN algorithm (details in Section 3.2.).

Step 3: Record results and calculate prediction error.

\section{Forecasting algorithms}

This chapter is mainly committed to two algorithms: AR and BP-ANN, for that AR-ANN is based on the previous two.

\subsection{AR forecasting algorithms}

AR method is a time series method which bases on the assumption that there is an internal structure of autocorrelation in data. AR model can be used for stationary processes and it forecasts the next time point value by fixing the order and the parameters of the model. Since load can be greatly influenced by weather conditions and some social factors, time series method maintains its stability in short period of time. AR method is usually applied for short-term or ultra-short-term load forecasting only. The three steps of AR algorithm:

Step 1: Data preprocessing. Difference processes are needed for those short-term load time series with inadequate stability. The second difference is usually enough. After that, calculate the mean value and variance of the time series and standardize it.

Step 2: Fix the order and estimate the parameters of the model.

The AR model is illustrated in equation (3).

$$
Y_{t}=c+\phi_{1} Y_{t-1}+\phi_{2} Y_{t-2}+\ldots+\phi_{p} Y_{t-p}+\varepsilon_{t}
$$


Where the process $\varepsilon_{t}$ is white noise; $C$ represents a constant related to the model and its default value is $0 . \phi_{1}, \ldots, \phi_{p}$ are the parameters of each order. The establishment of AR model is to fix $\varepsilon_{t}$, the order $p$ and the parameters $\phi_{p} . \varepsilon_{t}$ usually takes its mean value 0 ; order $p$ can be estimated by plotting the partial autocorrelation functions and then considering those functions for the residuals of the model. AIC (Akaike Information Criterion) [22] is applied for finding $p$. Finally the values of the parameters are calculated to minimize the error term.

Step 3: Calculate the forecasting value $Y_{t}$ from the AR model with inverse difference to replace part of the training samples of BP-ANN.

\subsection{BP-ANN Algorithm for multi-node load forecasting}

BP-ANN is an approach in supervised learning inspired by biological neural networks. The actual numerical weights assigned to element inputs are determined by matching historical data to desired outputs for several times in a pre-operational "training session". BP-ANN is a non-linear method, contrast to AR, and it can process massive parallel computing with multiple sources of data[23], which makes it more popular than AR and can be applied to longer term forecasting. However the results of BP-ANN can be affected by the selection of similar days in pre-day load forecasting.

Figure 3 shows the structure of Artificial Neural Network arranged in three layers, one input layer, one hidden layer and one output layer. Each segment in Figure 3 represents a non-linear function to its left side element, which is composed by a linear part of layer weights and a non-linear part of activation function. The linear function between the input layer I and the hidden layer J is shown in equation (4).

$$
y_{i}=f\left(\sum_{j} w_{i j} x_{j}\right)
$$

Where $y_{i}$ is the output of node $i ; x_{j}$ is the output of the upper node $j ; w_{i j}$ is the layer weight form node $i$ to node $j$; $f$ is the activation function.

We have a similar linear function between the hidden layer $\mathrm{J}$ and the output layer $\mathrm{K}$ where the corresponding weight is $w_{j k}$ in Figure 3.

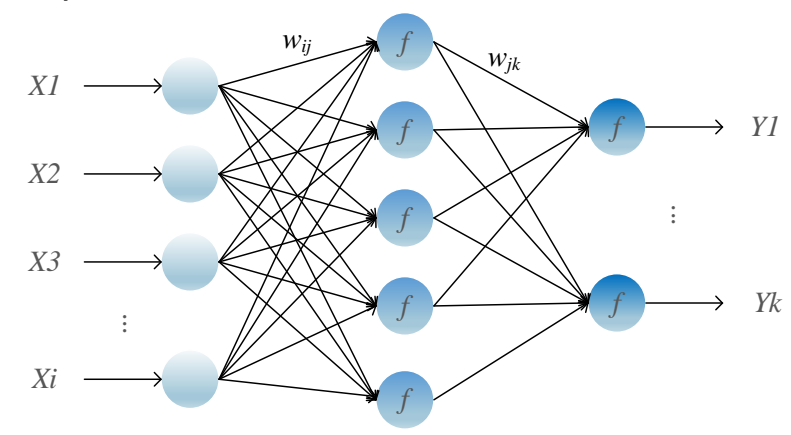

Inputs Input Layer I Hidden layer J Output Layer K Outputs

Figure 3. Structure of ANN

There are a number of common activation functions. This paper chooses a standard log-sigmoid function (also known as a logistic function), given by (5). 


$$
y_{i}=\frac{1}{1+\exp \left(-\sum_{j} w_{i j} x_{j}\right)}
$$

With the architecture of ANN in Figure 3, the task of BP is to find the values of weights. In short, the process of BP-ANN can be summarized in three parts: data preprocessing, data training and data forecasting. For the time efficiency of big data processing, the ANN applied in this paper is of three layers and the number of hidden nodes is determined by Kolmogorov's Theorem [24]. Data training part iterates the local gradients of the layers and the weight vectors until the MSE (minimum square error) reaches the acceptable range or the maximum number of iterations is reached, so that the weights are corrected. After that the parameters of ANN structure are determined, data forecasting part calculates the normalized output from the normalized input and then gets final forecasting results by a reverse of normalization process.

The flow chart of BN-ANN is presented in Figure 4. In particular, for multi-node load forecasting, the training data is defined to be the all types of data of the same time on the similar day. Thus the forecasting data is of the corresponding type. The complete BP-ANN multi-node load forecasting process is similar to Figure 2, except that the algorithm should be BP-ANN and it needs no pre-day load.
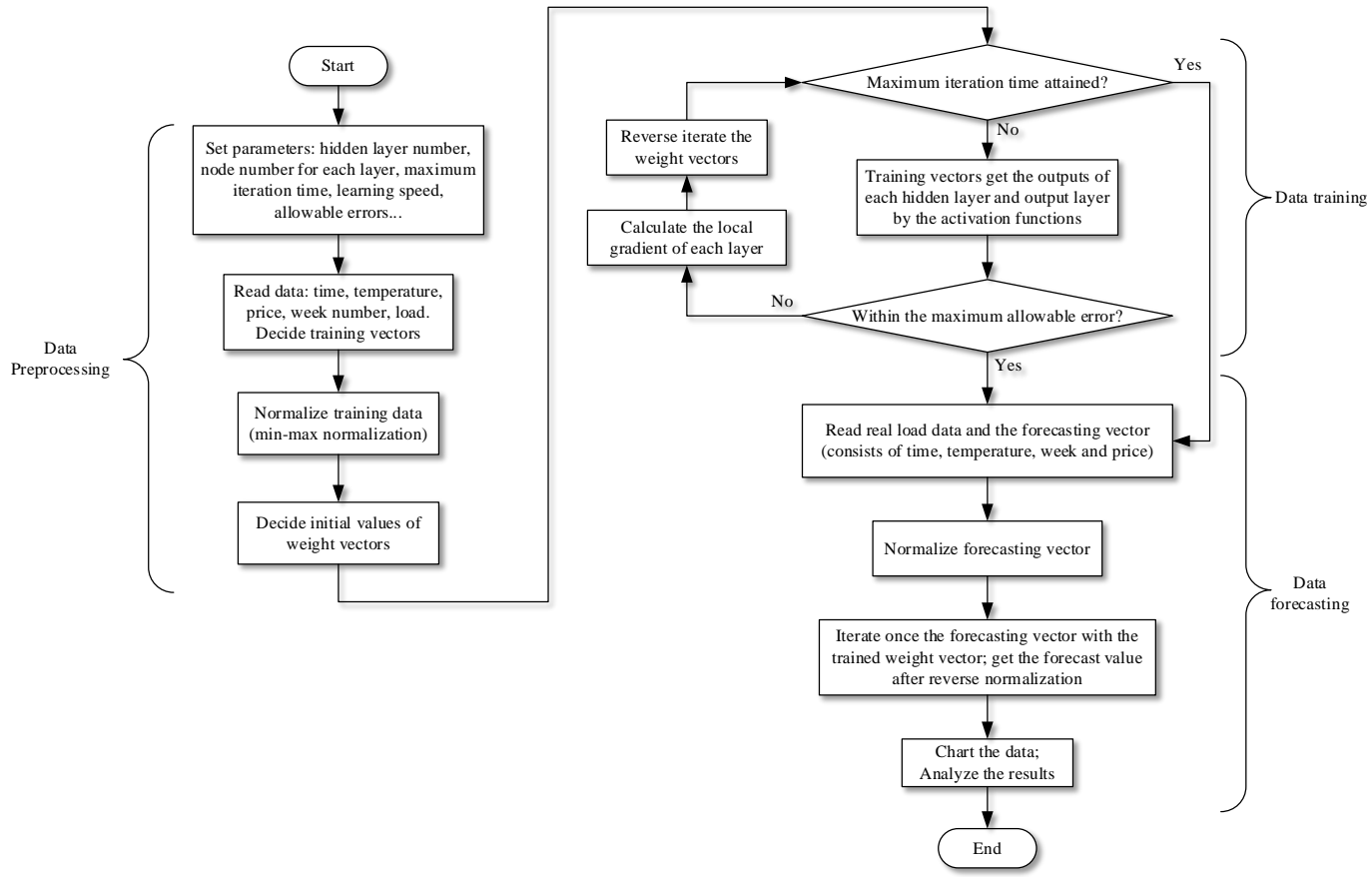

Figure 4. Flow chart of BP-ANN

\section{Simulation tests and results}

According to formula (1) and (2) in Section 2, multi-node load forecasting is the outcome of linear superposition of single-node load forecasting results. Since the errors in linear calculations are cumulative, we test the accuracy of AR-ANN algorithm by comparing the single-node load forecasting results of AR-ANN and BP-ANN, which is test 1.

Test 2 tries to compare the traditional bottom-up method with global load forecasting (regard the whole load as a single node) by both using AR-ANN algorithm. This test can also verify the application of AR-ANN algorithm to this type of multi-node load forecasting.

Finally in test 3, AR-ANN is applied to the top-down load forecasting case and compared with 
BP-ANN algorithm, to validate the advantages of AR-ANN and top-down method in multi-node load forecasting.

These three tests which are implemented on MATLAB include the load forecasting of holidays like $1^{\text {st }}$ January and ordinary working days. The accuracy of the results is reflected by maximum relative error, average relative error and root mean square error.

\subsection{Test 1: AR-ANN and BP-ANN for single-node load forecasting}

Today the big data with complex structures and giant size[25] updates its database and its volume quickly[26]. The big data load forecasting based on AR-ANN can utilize the latest measured data as AR in AR-ANN method can take advantage of the latest updated weather data to forecast the load.

Based on the pre-day forecasting, we forecast the 24 points of the forecasting day (1 point/hour). While adding the measured load data into the algorithm and forecasting the rest points of the day, there are 24 situations which correspond to different numbers of points to forecast.

The data comes from PJM Markets and Operations Database[27], and the load of the region run by AP Company is chosen to be the data for the whole load forecasting. The special forecasting day is $1^{\text {st }}$ January 2015 and its similar day is $1^{\text {st }}$ January 2014. The ordinary forecasting day sets to be $13^{\text {th }}$ January 2015 (Tuesday) with its similar day $6^{\text {th }}$ January 2015, Tuesday. PJM provides the hourly load (24 values/day) with the electricity price. We also obtain the weather data (the highest and lowest temperatures) of the load center for the last two years.

Considering the parameter setting, we take pre-day load forecasting for example: the node number for the input layer of ANN is 96 (); node number for the hidden layer is 193 (); the output layer has 24 nodes; maximum iteration number is 1000 and maximum allowable error is 0.001 .

BP-ANN and AR-ANN are respectively applied to forecasts these 24 situations mentioned before. The forecasting results of the special day (Figure 5 - Figure 7 ) and the ordinary day (Figure 8 - Figure 10) show that in most cases AR-ANN is more accurate than BP-ANN with smaller maximum relative error, smaller average relative error and smaller root mean square error. This means that for the most widely used single-node load forecasting, AR-ANN improves the traditional BP-ANN method with smaller errors for pre-day load forecasting. Even under the conditions of data update, AR-ANN adjusts well and maintains its advantages.

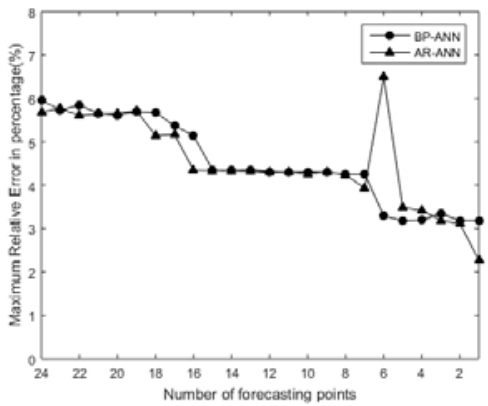

Figure 5. Comparison of MRE (maximum relative error) of load forecast on 01/01/2015 (special day) under multiple scenarios

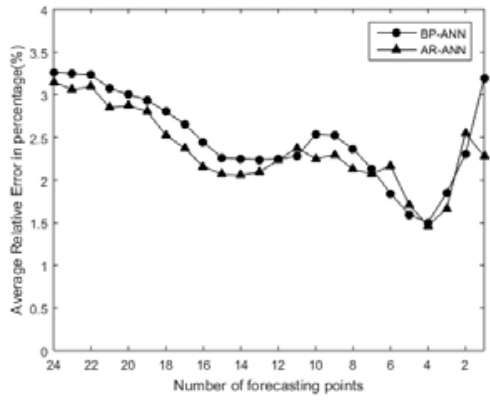

Figure 6. Comparison of ARE (average relative error) of load forecast on 01/01/2015 (special day) under multiple scenarios 


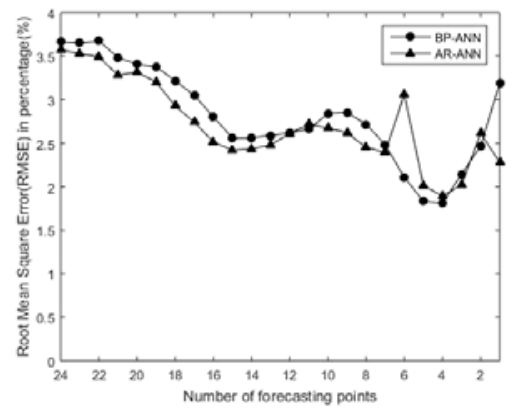

Figure 7. Comparison of RMSE of load forecast on 01/01/2015 (special day) under multiple scenarios

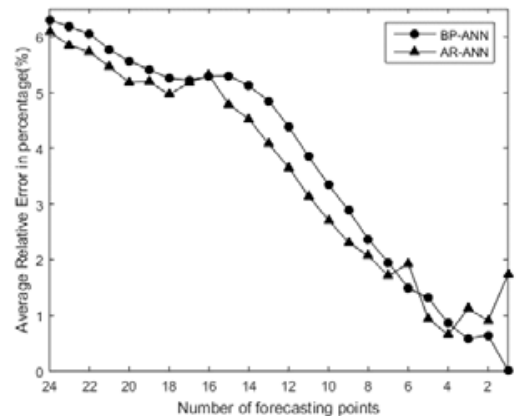

Figure 9. Comparison of ARE of load forecast on 13/01/2015 (ordinary day) under multiple scenarios

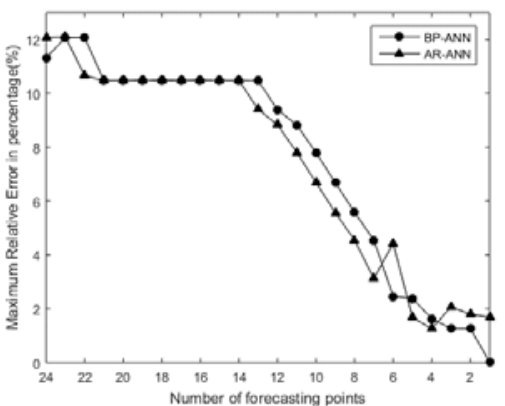

Figure 8. Comparison of MRE of load forecast on 13/01/2015 (ordinary day) under multiple scenarios

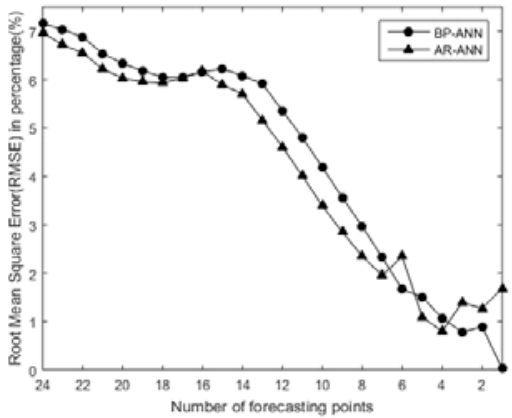

Figure 10. Comparison of RMSE of load forecast on 13/01/2015 (ordinary day) under multiple scenarios

\subsection{Test 2: AR-ANN for bottom-up multi-node load forecasting}

The data needed for load forecasting are possible to be in different databases [28]. Big data based load forecasting can be carried out by distributed methods with divide and conquer techniques [25]. Bottom-up multi-node load forecasting is exactly one of the distributed applications in the context of big data.

Test 2, based on the first test, forecasts the load from bottom to top. The "whole" is the PJM-WEST part which includes AP region. PJM-WEST has 8 nodes in total and AP region is one of those. The other parameters of test 2 are the same as the first one.

Figure 11 and Figure 12 give the forecasting results; table 2 and table 3 show the prediction errors of the special day and the ordinary day. We can see from the prediction errors that AR-ANN algorithm is effective for bottom-up multi-node load forecasting and this kind of load forecasting is slightly better than global load forecasting. Even though the results might be sometimes close to each other, multi-node forecasting consumes relatively less time by adapting the technique of distributed computing. In actual applications, AR-ANN multi-node load forecasting algorithm can not only detective the load variation of different nodes in distribution networks with accuracy, but also shorten the forecasting time by predicting local loads. 


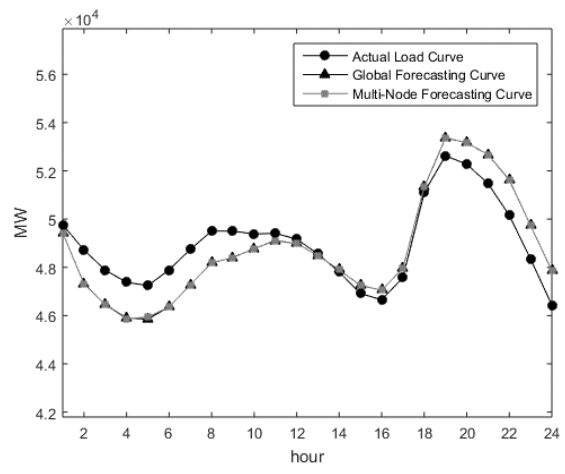

Figure 11. Comparison of different forecasting methods on 01/01/2015 (special day) in PJM-WEST

Table 2. Forecast statistics of Test 2 on a special day ( $1^{\text {st }}$ January 2015)

\begin{tabular}{|c|c|c|}
\hline Type of error & $\begin{array}{c}\text { AR-ANN } \\
\text { global } \\
\text { forecast }\end{array}$ & $\begin{array}{c}\text { AR-ANN } \\
\text { multi-node } \\
\text { forecast }\end{array}$ \\
\hline $\begin{array}{c}\text { Maximum } \\
\text { relative error } \\
\text { (MRE) }\end{array}$ & $3.50 \%$ & $3.33 \%$ \\
\hline $\begin{array}{c}\text { Average } \\
\text { relative error } \\
\text { (ARE) }\end{array}$ & $1.95 \%$ & $1.84 \%$ \\
\hline RMSE & $2.29 \%$ & $2.17 \%$ \\
\hline
\end{tabular}

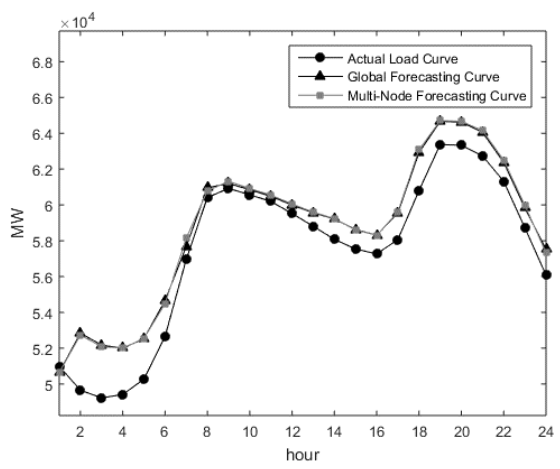

Figure 12. Comparison of different forecasting methods on 13/01/2015 (ordinary day) in PJM-WEST

Table 3. Forecast statistics of Test 2 on an ordinary day $\left(13^{\text {th }}\right.$ January 2015$)$

\begin{tabular}{|c|c|c|}
\hline Type of error & $\begin{array}{c}\text { AR-ANN } \\
\text { global } \\
\text { forecast }\end{array}$ & $\begin{array}{c}\text { AR-ANN } \\
\text { multi-node } \\
\text { forecast }\end{array}$ \\
\hline $\begin{array}{c}\text { Maximum } \\
\text { relative error } \\
\text { (MRE) }\end{array}$ & $6.41 \%$ & $6.20 \%$ \\
\hline $\begin{array}{c}\text { Average } \\
\text { relative error } \\
\text { (ARE) }\end{array}$ & $2.35 \%$ & $2.39 \%$ \\
\hline RMSE & $2.89 \%$ & $2.89 \%$ \\
\hline
\end{tabular}

\subsection{Test 3: Top-Down multi-node load forecasting}

In the environment of big data, the quick access between each database is not ideal [29] due to the factors like different permissions, dissimilar data structures and interface instability. With the developing size of distribution networks and higher density of measurements, missing data and abnormal data caused by terminal hardware failure increase. These lead to a growing demand for the efficiency of load forecasting. Thus this paper proposes a top-down method for multi-node load forecasting.

The data of test 3 comes from the SCADA system of Heze city, Shandong Province. The load of "City Line I" (35 kV) is considered as the "whole", and its "Distribution Transformer Line I" (DTI) carries the load as one of the "nodes". Since not all the lines of the DTI have measuring devices, we forecast the load of these non-measure lines with the help of "whole" load. In this test the special day chosen is the $15^{\text {th }}$ August 2015(Saturday) with its similar day the $8^{\text {th }}$ of the same month and same year; the ordinary forecasting day for test 3 is the $11^{\text {th }}$ August (Tuesday) in the same year and its similar day is the $4^{\text {th }}$. The measured load data of "City Line I" contains all the points including those of the pre-day, while "DTI" provides only the data of the similar day. Combing these data with the TOU (Time Of Use) price policy of Heze city and its extreme temperatures of each day, we construct a similar ANN structure to test 1 with the same parameters to forecast all the 24 load points ( 1 point/hour) of "DTI" on the $15^{\text {th }}$ August by using AR-ANN algorithm and BP-ANN algorithm respectively.

The forecasting load curve of two methods with the actual load curve are illustrated in Figure 13 and Figure 14. The average relative error of AR-ANN is 7.96\%, compared to $8.36 \%$ of BP-ANN (in Table 4). Thus top-down AR-ANN multi-node load forecasting produce better results than direct BP-ANN forecasting. Though with the reduce of user number, the increase of random factors and the occurrence of some special circumstances, the results might turn to be deviated like the ones of $20 \mathrm{~h}$ and $21 \mathrm{~h}$ in the 
test; the relative error remains within $10 \%$ for the most cases.

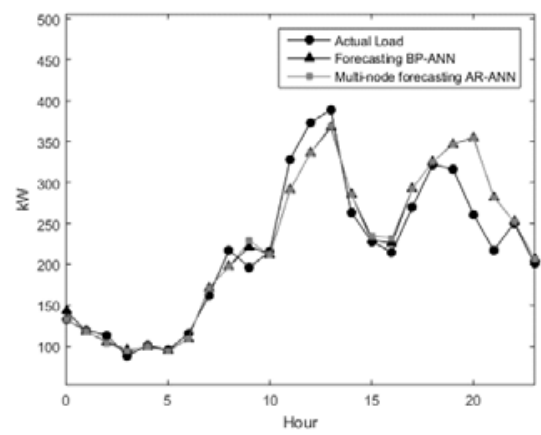

Figure 13. Comparison between forecast load and actual load of "DTI" of "City Line I" on $15 / 08 / 2015$ (special day)

Table 4. Forecast statistics of Test 3 on a special day (15 ${ }^{\text {th }}$ August 2015, Saturday)

\begin{tabular}{|c|c|c|}
\hline Type of error & $\begin{array}{c}\text { BP-ANN } \\
\text { load } \\
\text { forecast }\end{array}$ & $\begin{array}{c}\text { AR-ANN } \\
\text { multi-node } \\
\text { forecast }\end{array}$ \\
\hline $\begin{array}{c}\text { Maximum } \\
\text { relative error } \\
\text { (MRE) }\end{array}$ & $35.99 \%$ & $35.78 \%$ \\
\hline $\begin{array}{c}\text { Average relative } \\
\text { err(ARE) }\end{array}$ & $8.36 \%$ & $7.96 \%$ \\
\hline RMSE & $11.63 \%$ & $11.59 \%$ \\
\hline
\end{tabular}

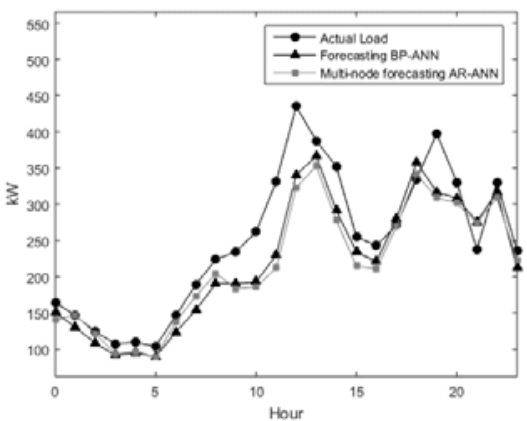

Figure 14. Comparison between forecast load and actual load of "DTI" of "City Line I" on 11/08/2015 (ordinary day)

Table 5. Forecast statistics of Test 3 on an ordinary day (1thAugust 2015, Tuesday)

\begin{tabular}{|c|c|c|}
\hline Type of error & $\begin{array}{c}\text { BP-ANN } \\
\text { load } \\
\text { forecast }\end{array}$ & $\begin{array}{c}\text { AR-ANN } \\
\text { multi-node } \\
\text { forecast }\end{array}$ \\
\hline $\begin{array}{c}\text { Maximum } \\
\text { relative error } \\
\text { (MRE) }\end{array}$ & $34.75 \%$ & $36.04 \%$ \\
\hline $\begin{array}{c}\text { Average } \\
\text { relative error } \\
\text { (ARE) }\end{array}$ & $14.23 \%$ & $12.83 \%$ \\
\hline RMSE & $15.88 \%$ & $15.73 \%$ \\
\hline
\end{tabular}

The results above show that AR part of AR-ANN method has quick access to the nearest database to forecast the "whole" load, and also forecasts the "node" load by their proportion. Even in the case of lack of data, the "node" load can be forecasted by AR-ANN method with the help of the "whole" database, and therefore facilitates different kinds of system analysis.

\section{Conclusion}

This paper proposes a novel algorithm for multi-node load forecasting, AR-ANN, which combines the advantages of AR time series method and BP-ANN non-linear algorithm. The single-node load forecasting in test 1 shows that in most cases AR-ANN obtains smaller errors than BP-ANN method; when applying to bottom-up multi-node load forecasting, this algorithm can shorten the processing time and reduce errors in some cases compared to global load forecasting.

A backward top-down method for multi-node load forecasting is also presented in this paper and tested by AR-ANN algorithm. The results show the possibility of load forecasting by AR-ANN when there is no access to some of the databases and/or a lack of data. Meanwhile, the inaccuracy of some forecasting points appeared in the test gives the space of further improvement.

This top-down short-term load forecasting algorithm, in conclusion, shows some advantages in forecasting precision and processing time efficiency. Furthermore, it can be utilized in the distribution network load forecasting to provide data for next step $\mathrm{N}-1$ contingency analysis and the study emergency power supply. 


\section{References}

1. K. Nose-Filho, A. D. P. Lotufo, and C. R. Minussi, "Short-term multinodal load forecasting using a modified general regression neural network," Power Delivery, IEEE Transactions on, 26, 2862-2869 (2011)

2. X. Han, L. Han, H. Gooi, and Z. Pan, "Ultra-short-term multi-node load forecasting-a composite approach,” Generation, Transmission \& Distribution, IET, 6, 436-444 (2012)

3. Z. Wang, C. Guo, and Y. Cao, "A new method for short-term load forecasting integrating fuzzy-rough sets with artificial neural network," in Power Engineering Conference, 2005. IPEC 2005. The 7th International, 1-173 (2005)

4. S. Khatoon and A. Singh, "Analysis and comparison of various methods available for load forecasting: An overview, ” CIPECH, 2014 Innovative Applications of, 243-247 (2014)

5. H. S. Hippert, C. E. Pedreira, and R. C. Souza, "Neural networks for short-term load forecasting: A review and evaluation,” Power Systems, IEEE Transactions on, 16, 44-55 (2001)

6. J. Wang and Q. Zhu, "Short-term electricity load forecast performance comparison based on four neural network models, " CCDC, 2928-2932 (2015)

7. X. Wang and L. Meng, "Ultra-short-term load forecasting based on EEMD-LSSVM," (2015)

8. M. Zhe and S. Qin, "Short term load forecasting based on ESPRIT integrated algorithm," (2015)

9. B. Lu, S. Zhao, Y. Tian, Y. Yang, B. Li, X. Chen, et al., "Mid-long term electricity consumption forecasting based on improved NGM $(1,1, \mathrm{k})$ gray model," Power System Protection and Control, 98-103 (2015)

10. W. Mo, B. Zhang, H. Sun, and Z. Hu, "Method to select similar days for short-term load forecasting," Journal Tsinghua University, 44, 106-109 (2004)

11. H. Lin, J. Liu, Z.-f. Hao, F.-f. Zhu, and G.-c. Wu, "Short-term load forecasting for holidays based on the similar days' load modification,” Relay, 7, 47-51 (2010)

12. C. Li, X. Li, R. Zhao, J. Li, and X. Liu, "A Novel Algorithm of Selecting Similar Days for Short-term Power Load Forecasting [J]," Automation of Electric Power Systems, 9, 018 (2008)

13. Z.-l. Yang, Y. Tian, G.-t. Zhang, and K.-y. Lin, "Nonlinear Theoretical Foundation and Improvement of Similar Days Method for Short Term Load Forecasting [J]," Power System Technology, 6, 014 (2006)

14. G. Ye, Y. Luo, Y. Liu, and H. Jin, "Research on Method of Power System Load Forecasting Based on ARMA Model [J]," Electronic Technology (2002)

15. Z. Zhou, J. Li, and X. Zhang, "The application of ANN in middle term load forecasting of power system", Proceedings of the CSU-EPSA, 36-39 (2003)

16. J. He, G. Wei, L. Xiong, "Fuzzy improvement of linear regression analysis for load forecasting [J]," East China Electric Power, 11, 21-23 (2003)

17. J. Lian, H. Liu, H. Xie, X. Gong, and X. Xu, "Classified load balancing algorithm based on prediction mechanism," Computer Engineering and Applications, 67-71,98 (2015)

18. M. Zhang, H. Bao, L. Yan, J.-p. Cao, and J.-g. Du, "Research on processing of short-term historical data of daily load based on Kalman filter [J]", Power System Technology, 10, 009, 200

19. G. Chen, F. Yan, X. Gong, and Y. Wang, "State estimate based on parameter-optimized least square support vector machines," Power System Protection and Control, 39, 83-88 (2011)

20. J.-f. Zhang, Y.-a. Wu, and J.-j. Wu, "Application of gray system theory in load forecasting [J]," Electric Power Automation Equipment, 5, 005 (2004)

21. J. W. Taylor and P. E. McSharry, "Short-term load forecasting methods: An evaluation based on European data," Power Systems, IEEE Transactions on, 22, 2213-2219 (2007)

22. Y. Guo, "The methods of spectrum density estimation and prediction of multidimensional ARMA(p,q) models" (Southwest Jiaotong University, 2008)

23. C. Lv, "Short-term load forecasting based on BP artificial neural network" (Huazhong University of Science and Technology, 2007)

24. Y. Shao, "Research on electric power system short-term load forecasting using a neural network",(Harbin University of Science and Technology, 2005) 
25. S. Zhang, B. Zhao, F. Wang, and D. Zhang "Short-term power load forecasting based on big data," Proceedings of the CSEE, 37-42 (2015)

26. D. Wang and Z. Sun, "Big Data Analysis and Parallel Load Forecasting of Electric Power User Side," Proceedings of the CSEE, 3, 004 (2015)

27. PJM - Markets \& Operations. Available: http://www.pjm.com/markets-and-operations.aspx (2016)

28. K. Liu, W. Sheng, D. Zhang, D. Jia, L. Hu, and K. He, "Big data application requirements and scenario analysis in smart distribution network," Proceedings of the CSEE, 35, 287-293 (2015)

29. Y. Song, G. Zhou, Y. Zhu, L. Li, L. Wang, and D. Wang, "Storage Optimization and Parallel Processing of Condition Monitoring Big Data of Transmission and Transforming Equipment Based on Cloud Platform," Proceedings of the CSEE, 2, 001 (2015) 